K.V. Chunikhin

\title{
ON THE INFLUENCE OF THE LEVEL OF AN EXTERNAL MAGNETIC FIELD AND THE LENGTH ON THE MAGNETIC MOMENT OF CYLINDRICAL CORES
}

Purpose. Analysis of inhomogeneous magnetization of long cylindrical permalloy 50N cores by a uniform constant magnetic field and the influence of length and field level on their magnetic moment. Methodology. The magnetostatic field of a non-uniformly magnetized in a uniform magnetic field long cylindrical core of an electromagnet of a spacecraft control system is considered. To calculate this field, a transformation of the integral equation with respect to the density of fictitious magnetic charges, as well as an iterative algorithm for its numerical solution, are proposed. Results. The convergence of the algorithm and the fact that the magnetic moment of the core depends heavily on its length and the level of the external magnetic field is shown. We have made an analysis of the influence of the length of a permalloy $50 \mathrm{~N}$ core in the entire range of the magnetization curve and the level of a uniform external magnetic field on the axial projection of the magnetic moment of the core. Originality. The use of an almost equal distribution of the axial projection of the resulting magnetic field in the cross sections of the greater part of the cylindrical core and its division into cylindrical elements can significantly reduce the order of the system of algebraic equations approximating the integral equation for the surface density of fictitious magnetic charges for its numerical solution. Practical value. Recommendations regarding the level of the external field created by the electromagnet coil, the increase of the magnetic moment in cases of long cores and the choice of the number of cylindrical elements depending on the length of the core are given. References 14, tables 1, figures 5.

Key words: electromagnet, spacecraft control system, non-uniformly magnetized core, integral equation, fictitious magnetic charge, magnetization curve, magnetic moment of the core.

Рассмотрено магнитостатическое поле неоднородно намагниченного в однородном магнитном поле длинного цилиндрического сердечника электромагнита системы управления космическим аппаратом. Для расчета этого поля предложены преобразование интегрального уравнения относительно плотности фиктивных магнитных зарядов, а также итерационный алгоритм его численного решения. Сделан анализ влияния длины сердечника из пермаллоя $50 Н$ во всем диапазоне кривой намагничивания и уровня внешнего магнитного поля на осевую проекцию магнитного момента сердечника, а также даны практические рекомендации относительно уровня внешнего поля, создаваемого катушкой электромагнита, и увеличения магнитного момента в случаях длинных сердечников. Библ. 14, табл. 1, рис. 5.

Ключевые слова: электромагнит, система управления космическим аппаратом, неоднородно намагниченный сердечник, интегральное уравнение, фиктивный магнитный заряд, кривая намагничивания, магнитный момент сердечника.

Introduction. To control the spacecraft, DC electromagnets consisting of a coil and a long cylindrical core of a material with high magnetic permeability are used [1]. Dimensions, winding data and materials can be determined and selected based on the calculation of the magnetic field generated by the electromagnet. Such an electromagnet must have a certain magnetic moment, the main part of which is provided by the core [2]. In the well-known works, for the calculation of the magnetostatic field of the cores the methods of demagnetization coefficients $[2,3]$ and integral equations $[1,4,5]$ are developed. To determine the demagnetization coefficients, it is necessary to carry out experiments, and numerical solutions of integral equations were obtained under the assumption of a constant magnetic permeability of the core material $[1,4]$. In [5], the calculations were performed under the condition of relatively small changes in the magnetic permeability along the core volume.

The relevance of this paper lies in the fact that in well-known works the magnetization of cylindrical cores is insufficiently investigated in conditions of large changes in magnetic permeability and levels of an external magnetic field, which makes it difficult to design electromagnets for spacecraft control systems.

The goal of the work is analysis of inhomogeneous magnetization of long cylindrical permalloy $50 \mathrm{~N}$ cores by a uniform constant magnetic field and the influence of length and field level on their magnetic moment.
Transformation of the original integral equation taking into account the peculiarities of the magnetization of the core. Consider a cylindrical core of length $b$ and of radius $R$ located in an unbounded nonmagnetic and nonconducting space coaxially with an external constant uniform magnetic field of strength $\vec{H}_{0}$ (Fig. 1).

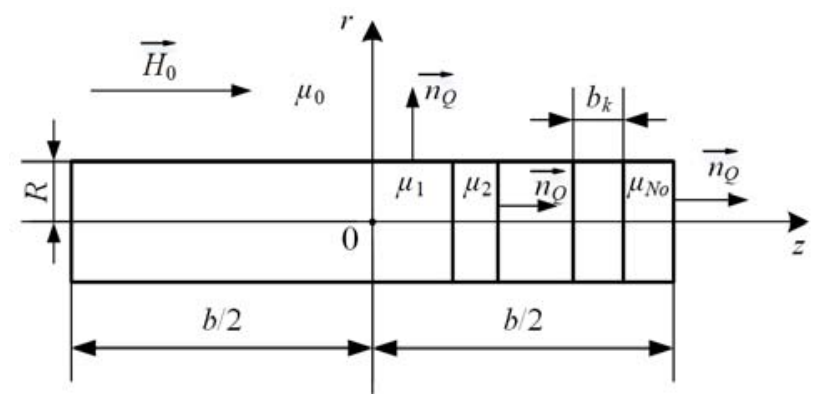

Fig. 1. Meridian section of the cylindrical core

The strength of the resulting magnetic field is represented as $[6,7]$ :

$$
\vec{H}=\vec{H}_{0}+\vec{H}_{m}
$$

where $\vec{H}_{m}$ is the magnetic field strength due to the magnetic properties of the core («demagnetizing» field [2]). 
The magnetic field $\vec{H}$ is plane-meridian, and the vector field $\vec{H}_{m}$ is potential and related to the scalar potential $\varphi_{m}$ by

$$
\vec{H}_{m}=-\operatorname{grad} \varphi_{m} .
$$

Using the electrostatic analogy $[6,8]$, we represent $\varphi_{m}$ в in the following form $[9,10]$ :

$$
\varphi_{m}(Q)=\frac{1}{\pi \mu_{0}} \int_{l} \frac{\sigma_{m}(M) r_{M} K(k)}{\sqrt{\left(z_{Q}-z_{M}\right)^{2}+\left(r_{Q}+r_{M}\right)^{2}}} d l_{M},
$$

where $l, d l_{M}$ are the contour of the meridian section and its element with the center in the point $M ; Q, M \in l$ are the observation point and the point with current coordinated; $\sigma_{m}(M)$ is the surface density of fictitious magnetic charges; $\mu_{0}$ is the magnetic constant; $K(k)$ is the complete elliptic integral of the first kind of module $k$ [11];

$$
k=2 \sqrt{\frac{r_{Q} r_{M}}{\left(z_{Q}-z_{M}\right)^{2}+\left(r_{Q}+r_{M}\right)^{2}}} ;
$$

$r_{Q}, r_{M}$ and $z_{Q}, z_{M}$ are the radial and axial cylindrical coordinates of points $Q$ and $M$.

Since the core material is isotropic, the relationship between $\vec{H}$ and the magnetization $\vec{J}$ is determined by the known dependence

$$
\vec{J}=\left[\mu_{r}(H)-1\right] \vec{H},
$$

where $\mu_{r}(H)$ is the relative magnetic permeability.

To take into account the inhomogeneity of magnetization, we replace the nonlinear magnetized medium of the core with a piecewise homogeneous medium, which consists of $2 N_{o}$ homogeneous cylindrical elements with absolute magnetic permeability $\mu_{k}$, $k=\overline{1, N_{o}}$, having a length $b_{k}$, and $\sum_{k=1}^{N_{o}} b_{k}=b / 2$ (Fig. 1). Such a replacement allows to neglect volume fictitious magnetic charges and limit the definition of $\sigma_{m}$. In this case, the integral equation for $\sigma_{m}$ takes the form [12]:

$$
\begin{gathered}
\sigma_{m}(Q)-\frac{\lambda_{\mu}}{\pi} \int_{l} \sigma_{m}(M)\left[S(Q, M)-S\left(Q, M^{\prime}\right)\right] d l_{M}= \\
=2 \mu_{0} \lambda_{\mu} H_{0 n}(Q),
\end{gathered}
$$

where $l$ is the contour of the meridian section of the core in the first quarter of the coordinate plane $z$ Or; $l=l_{1}+$ $+l_{2}+l_{3} ; l_{1,2}$ is the contour of the side and end surfaces; $l_{3}=\sum_{k=1}^{N_{o}-1} l_{k}^{c}, l_{k}^{c}$ is the boundary between $k$ and $k+1$ cylindrical elements in the calculation region; $H_{0 n}(Q)$ is the normal projection of $\vec{H}_{0}$ for $Q \in l$;

$$
\lambda_{\mu}=\left\{\begin{array}{l}
\left(\mu_{k}-\mu_{0}\right) /\left(\mu_{k}+\mu_{0}\right), Q \in l_{1} \cup l_{2} ; \\
\left(\mu_{k}-\mu_{k+1}\right) /\left(\mu_{k}+\mu_{k+1}\right), Q \in l_{3}
\end{array}\right.
$$

$M$ ' is the point symmetric to point $M$ relative to the $r$-axis.

At $Q \in l_{1}$ the first term of the kernel of the equation (5) $S(Q, M)=\frac{k}{2} \sqrt{\frac{r_{M}}{r_{Q}^{3}}}\left[K(k)+\frac{1}{k^{\prime 2}}\left(\frac{r_{M}+r_{Q}}{2 r_{M}} k^{2}-1\right) E(k)\right]$, and at $Q \in l_{2} \cup l_{3}-S(Q, M)=\frac{z_{Q}-z_{M}}{4 \sqrt{r_{Q}^{3} r_{M}}} \frac{k^{3}}{k^{\prime 2}} E(k)$, where $E(k)$,

$k^{\prime}$ is the complete elliptic integral of the second kind of modulus $k$ and additional modulus of complete elliptic integrals, $k^{\prime}=\sqrt{1-k^{2}}$ [11]. The second term of the considered kernel $S\left(Q, M^{\prime}\right)$ is determined by the same formulas as $S(Q, M)$ if in them to replace the coordinates of the point $M$ by $M^{\prime}$.

The replacement of a non-uniformly magnetized core with a set of uniformly magnetized cylindrical elements was made on the basis of preliminary calculations, according to which at $b / R \geq 16$ the axial projection of $\vec{H}$ in the cross sections of the core along its entire length, except for small sections near the ends, is distributed almost uniformly. Considering this feature, as well as the well-known boundary condition about the jump of the normal projection of $\vec{H}_{m}$ on the boundary between two magnetized media [13], we assume that $\sigma_{m}$ is invariable for all $l_{k}^{c}$. Simplified by this assumption, equation (5) takes the following form:

$$
\begin{gathered}
\sigma_{m}(Q)-\frac{\lambda_{\mu}}{\pi} \int_{l-l_{3}} \sigma_{m}(M)\left[S(Q, M)-S\left(Q, M^{\prime}\right)\right] d l_{M}- \\
-\frac{\lambda_{\mu}}{\pi} \sum_{k=1}^{N_{o}-1} \sigma_{m}\left(M_{k}\right) \int_{l_{k}^{c}}\left[S(Q, M)-S\left(Q, M^{\prime}\right)\right] d l_{M}= \\
=2 \mu_{0} \lambda_{\mu} H_{0 n}(Q) .
\end{gathered}
$$

The system of algebraic equations, with the help of which the integral equations are solved, for equation (6) has a much smaller order.

An iterative algorithm for the numerical solution of a transformed integral equation. The iteration algorithm cycle consists of the following main blocks. In the first block, for some initial values of $\mu_{k}{ }^{(0)}$, we solve the integral equation (6). To do this, using the quadrature formula of rectangles, it was transformed into a system of algebraic equations of order $N(N$ is the total number of nodes of the spatial mesh, $N=N_{1}+N_{2}+N_{3}, N_{1}$ is the number of nodes per $l_{1}, N_{2}-$ per $l_{2}, N_{3}-\operatorname{per} l_{3}, N_{3}=N_{o}-1$ ). When calculating each integral of the sum in the third term of the left-hand side of (6), 100 nodes were taken, and to take into account the edge effect at the cylinder ends, a non-uniform mesh was used. The obtained system of algebraic equations was solved by a direct method based on inverting the matrix of the left-hand sides and further multiplying the inverse matrix by a column vector of the right-hand sides.

In the second block we find the radial and axial projections of $\vec{H}$

$$
\begin{gathered}
H_{r}(Q)=\frac{1}{2 \pi \mu_{0}} \int_{l} \sigma_{m}(M) S_{r}(Q, M) d l_{M}, \\
H_{z}(Q)=H_{0}+\frac{1}{2 \pi \mu_{0}} \int_{l} \sigma_{m}(M) S_{z}(Q, M) d l_{M},
\end{gathered}
$$

and then the module of $\vec{H}$ at each point inside the core. 
In formulas (7) and (8), the functions $S_{r}(Q, M)$ and $S_{z}(Q, M)$ are determined using the same expressions as the kernel of the integral equation (5), respectively, $Q \in l_{1}$ and $Q \in l_{2} \cup l_{3}$.

In the third block, we determine average over the volume of each cylindrical element the relative and absolute magnetic permeabilities

$$
\bar{\mu}_{k r}^{(j)}=\frac{2}{R^{2} b_{k}} \int_{S_{k}} r_{M} \mu_{r}(M) d S_{M}, \quad \bar{\mu}_{k}^{(j)}=\mu_{0} \bar{\mu}_{k r}^{(j)},
$$

where $S_{k}$ is the meridian area of the $k$-th cylindrical element; $j$ is the iteration number.

To determine $\mu_{r}$, we use the magnetization curve of permalloy $50 \mathrm{~N}$ [2]

$$
J(H)=a H /(H+c),
$$

from which with the help of a known connection between $\vec{B}, \vec{H}$ and $\vec{J}$ on the basis of the model of magnetization by molecular currents, we find

$$
\mu_{r}(H)=1+a /(H+c) \text {. }
$$

In dependencies (11), (12), shown using the logarithmic scale in Fig. $2(a, b), J, H$ are the modules of $\vec{J}$, $\vec{H} ; a, c$ are the constants, $a=1.25 \cdot 10^{6} \mathrm{~A} / \mathrm{m}, c=40 \mathrm{~A} / \mathrm{m}$. Then we take $\mu_{k}^{(j)}=\bar{\mu}_{k}^{(j)}$ and return to the first block of the algorithm. We continue iterations until the condition

$$
\left|\mu_{k}^{(j)}-\mu_{k}^{(j-1)}\right|<\Delta, j=1,2, \ldots, n_{i t}, k=\overline{1, N_{o}},
$$

where $\Delta$ is the specified discrepancy; $n_{i t}$ is the iteration number.
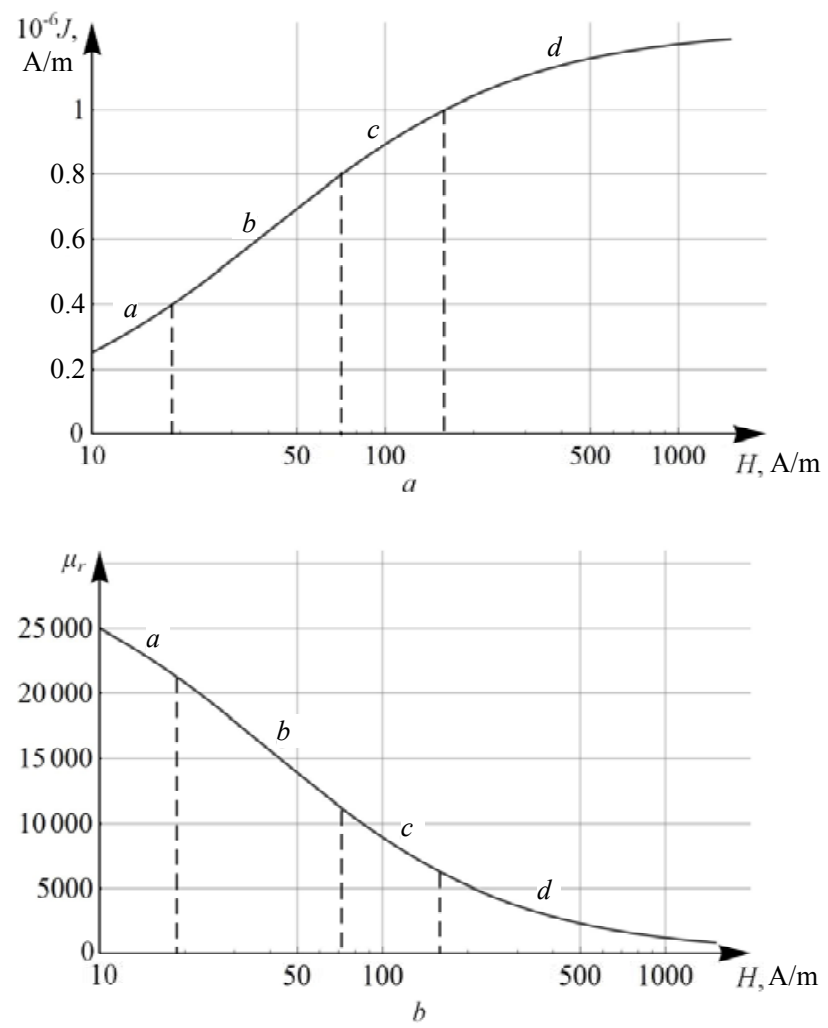

Fig. 2. Dependencies $J(H)-a$ and $\mu_{r}(H)-b$ for permalloy $50 \mathrm{~N}$

It is established that the iteration process converges to some values of $\mu_{k}{ }^{(i t)}$ for any $\mu_{k}{ }^{(0)}$, for which we have a numerical solution of (6). As explained below, the magnetic moment of the core is determined by the axial projection of the magnetization $J_{z}$. After determining $\sigma_{m}$ this projection can be calculated using formula (4), taking into account (8) and (12). The convergence of the iterations is illustrated by the curves in Fig. 3, constructed for the following initial data: $R=5 \mathrm{~mm}$ (this size is assumed to be the same in subsequent calculations); $b=330 \mathrm{~mm} ; z^{*}=z / b$. The values of $b_{k}$ are hereinafter assumed to be the same. We see that with increasing $N_{o}$, the distributions of $J_{z}$ converge. If it is necessary to clarify the influence of edge effects, the cylindrical elements at the edges of the core can be replaced by a set of ring elements. Note that in the work [5], when the integral equation regarding tangential projection of the magnetization was numerically solved in accordance with the recommendations of [14], the entire volume of the core was divided into ring elements.

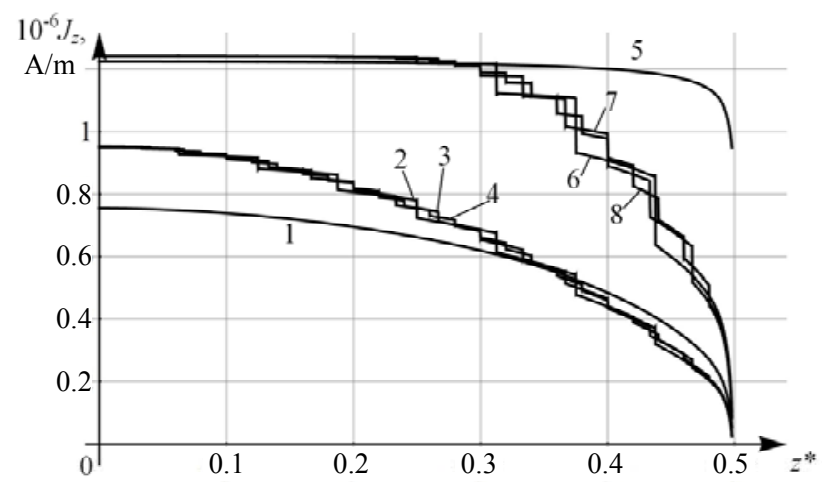

Fig. 3. Distribution of the axial projection of magnetization along the core axis at different $N_{o}$ for $H_{0}=1646.66 \mathrm{~A} / \mathrm{m}$ (curves 1-4) and $H_{0}=6586.62 \mathrm{~A} / \mathrm{m}$ (curves 5-8); for curves 1 and 5, 2 and 6, 3 and 7, 4 and 8 values of $N_{o}$ respectively equal to 1,8 , 15,25

The influence of the level of the external magnetic field and the length of the core on its magnetic moment. Due to the axial symmetry of the field, the magnetic moment vector $\vec{M}$ of the core under consideration has only an axial projection in cylindrical coordinates

$$
M_{z}=4 \pi \int_{S} r_{M} J_{z}(M) d S_{M},
$$

where $S$ is the part of the area of the meridian section in the positive half-plane $z>0$.

Table 1 shows the values of $M_{z}$ and the relative discrepancies $\xi$ between $M_{z}$ at $N_{o}=25$ (conventionally exact values) and $M_{z}$ at smaller $N_{o}$. It follows from the above data that to ensure $\xi \leq 1 \%$ for all considered levels of $H_{0}$ and values $b=80,165$ and $330 \mathrm{~mm}, N_{o}$ should be taken equal to 4,8 and 8 , respectively. Note that for a core with a length $80 \mathrm{~mm}$ with $N_{o}=1$ the value of $\xi \approx(1 \div 6) \%$ (obviously, this is also true for cores of not very different length).

Fig. 4, 5 show the influence of the level of the external field and the core length on the value of $M_{z}$ (values of $H_{0}$ are marked with dots on the abscissa axis in Fig. 4, and the corresponding values of $M_{z}$ are shown on the curves). Calculations show that at each point of the core, with increasing $H_{0}$ the strength of the resulting field always increases, but the magnetization depends on which 
part of the magnetization curve $H_{z}$ falls into (Fig. 2). In section $a$ of the $\mu_{r}(H)$ curve (Fig. 2,b), the magnetic permeability is maximum, which leads to large values of $H_{m}$ and, as a consequence, small values of $H$. In the same part of the initial magnetization curve (Fig. 2,a), the latter corresponds to small values of $J$. At $b=80 \mathrm{~mm}$, this leads to relatively small magnetic moments $M_{z}=0.5 \div 2.8 \mathrm{~A} \cdot \mathrm{m}^{2}$ (Fig. 4, curve 1; Fig. 5).

Table 1

Values at different $b, H_{0}, N_{o}$

\begin{tabular}{|c|c|c|c|c|c|}
\hline \multirow{2}{*}{$\begin{array}{c}b, \mathrm{~mm} ; \\
b / R\end{array}$} & \multirow{2}{*}{$N_{o}$} & \multicolumn{4}{|c|}{$H_{0}, \mathrm{~A} / \mathrm{m}$} \\
\hline & & 1646.66 & 3293.31 & 6586.62 & 9879.93 \\
\hline \multirow{7}{*}{$80 ; 16$} & 25 & 0.500 & 0.989 & 1.937 & 2.845 \\
\hline & 8 & 0.4998 & 0.9886 & 1.9342 & 2.8396 \\
\hline & $\xi, \%$ & 0.0392 & 0.0742 & 0.1348 & 0.1863 \\
\hline & 4 & 0.499 & 0.986 & 1.926 & 2.822 \\
\hline & $\xi, \%$ & 0.150 & 0.294 & 0.562 & 0.803 \\
\hline & 1 & 0.495 & 0.970 & 1.862 & 2.686 \\
\hline & $\xi, \%$ & 1.006 & 1.985 & 3.838 & 5.587 \\
\hline \multirow{7}{*}{$165 ; 33$} & 25 & 3.090 & 5.927 & 10.839 & 13.043 \\
\hline & 15 & 3.088 & 5.921 & 10.825 & 13.026 \\
\hline & $\xi, \%$ & 0.050 & 0.094 & 0.135 & 0.127 \\
\hline & 8 & 3.083 & 5.904 & 10.776 & 12.972 \\
\hline & $\xi, \%$ & 0.213 & 0.396 & 0.585 & 0.540 \\
\hline & 1 & 2.963 & 5.486 & 9.537 & 12.591 \\
\hline & $\xi, \%$ & 4.084 & 7.445 & 12.019 & 3.462 \\
\hline \multirow{7}{*}{$330 ; 66$} & 25 & 17.863 & 25.330 & 28.394 & 29.546 \\
\hline & 15 & 17.834 & 25.288 & 28.342 & 29.481 \\
\hline & $\xi, \%$ & 0.164 & 0.166 & 0.182 & 0.220 \\
\hline & 8 & 17.765 & 25.186 & 28.202 & 29.331 \\
\hline & $\xi, \%$ & 0.551 & 0.568 & 0.677 & 0.728 \\
\hline & 1 & 15.796 & 24.538 & 31.252 & 31.964 \\
\hline & $\xi, \%$ & 11.571 & 3.126 & 10.067 & 8.184 \\
\hline
\end{tabular}
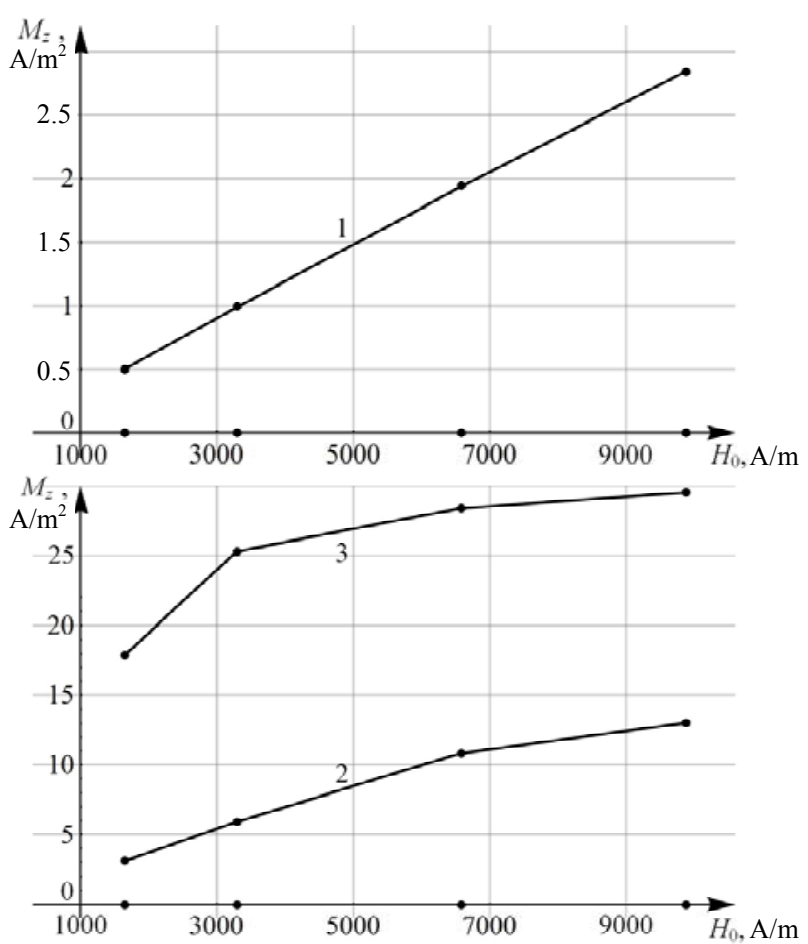

Fig. 4. Dependencies $M_{z}\left(H_{0}\right)$ at $N_{o}=25$ : curve $1-b=80 \mathrm{~mm}, 2-165 \mathrm{~mm}, 3-330 \mathrm{~mm}$
With an increase in the length of the core, we observe a significant increase in the magnetic moment (Fig. 4, curves 2, 3; Fig. 5), since the core points are magnetized either in all parts of the magnetization curve ( $b=165 \mathrm{~mm})$, or in sections $c, d(b=330 \mathrm{~mm})$ with significantly larger $J$ (Fig. 2,a). However, the growth of $M_{z}$ of cores of greater length with increasing $H_{0}$ slows down, since an increasing part of them is in a state of saturation. From Fig. 5 it follows that at $b / R=(33 \div 66)$, to achieve $M_{z} \leq 18 \mathrm{~A} \cdot \mathrm{m}^{2}, H_{0}=1646.66 \mathrm{~A} / \mathrm{m}$ is sufficient. For larger values of $M_{z}$, up to $25 \mathrm{~A} \cdot \mathrm{m}^{2}, H_{0}=3293.31 \mathrm{~A} / \mathrm{m}$ is required.

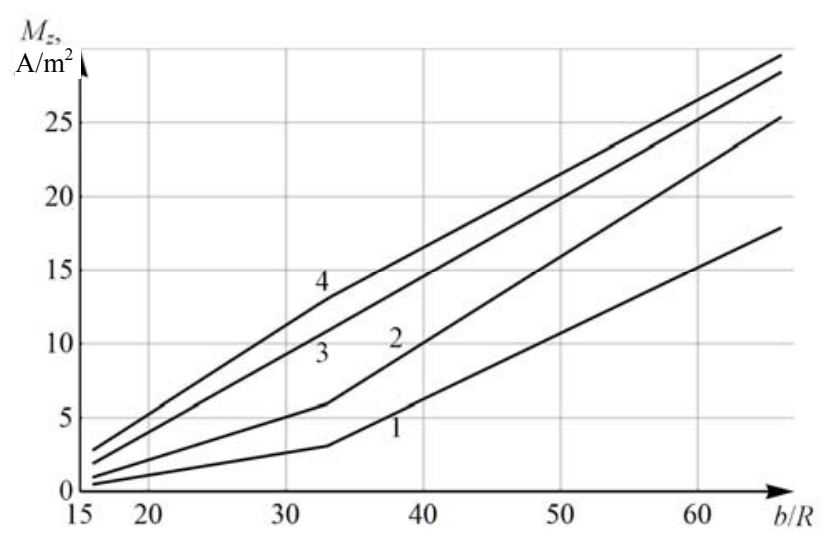

Fig. 5. Dependencies $M_{z}(b / R)$ at $N_{o}=25$ : curve $1-H_{0}=1646.66 \mathrm{~A} / \mathrm{m}, 2-3293.31 \mathrm{~A} / \mathrm{m}$ $3-6586.62 \mathrm{~A} / \mathrm{m}, 4-9879.93 \mathrm{~A} / \mathrm{m}$

\section{Conclusions.}

1. The choice of the sizes of the cylindrical cores of electromagnets of spacecraft control systems must be carried out on the basis of a given maximum value of the axial projection of the magnetic moment $M_{z}$ and the magnetization curve of the core material.

2 . The coil of an electromagnet should provide such levels of an external magnetic field at which the strength of the resulting magnetic field on the predominant part of the core is outside the saturation region of the magnetization curve and corresponds to a higher magnetization.

3. At $R=5 \mathrm{~mm}$, cores with a relative length of $b / R<33$ provide $M_{z} \leq 13 \mathrm{~A} \cdot \mathrm{m}^{2}$. In cases of $b / R>33$, an increase in $M_{z}$ can be achieved by increasing $b / R$ at certain levels of the external magnetic field, which do not lead to the saturation of a significant part of the core (Fig. 4, 5).

\section{REFERENCES}

1. Chadebec O., Rouve L.-L., Coulomb J.-L. New methods for a fast and easy computation of stray fields created by wound rods. IEEE Transaction on Magnetics, 2002, vol.38, no.2, pp. 517-520. doi: 10.1109/20.996136.

2. Kovalenko A.P. Magnitnye sistemy upravleniia kosmicheskimi letatel'nymi apparatami [Magnetic control systems for space vehicles]. Moscow, Mashinostroenie Publ., 1975. 248 p. (Rus).

3. Rozenblat M.A. Demagnetization factors for high permeability rods. Technical Physics, 1954, vol.24, no.4, pp. 637-661. (Rus).

4. Chen D.X., Pardo E., Sanchez A. Fluxmetric and magnetometric demagnetizing factors for cylinders. Journal of Magnetism and Magnetic Materials, 2006, vol.306, pp. 135-146. doi: 10.1016/j.jmmm.2006.02.235. 
5. Matiuk V.F., Osipov A.A., Streliukhin A.V. Modeling of the magnetic state of a ferromagnetic rod in longitudinal constant magnetic field. Technical Diagnostics and Non-Destructive Testing, 2011, no.1, pp. 20-27. (Rus).

6. Grinberg G.A. Izbrannye voprosy matematicheskoi teorii elektricheskikh $i$ magnitnykh iavlenii [Selected questions of mathematical theory of electric and magnetic phenomena] Moscow-Leningrad, Acad. of Sci. USSR Publ., 1948. 730 p. (Rus).

7. Tozoni O.V., Maergoiz I.D. Raschet trekhmernykh elektromagnitnykh polei [Calculation of three-dimensional electromagnetic fields]. Kiev, Tekhnika Publ., 1974. 352 p. (Rus).

8. Mikhailov V.M., Chunikhin K.V. On electrostatic analogy of magnetostatic field in inhomogeneous magnetized medium. Electrical engineering \& electromechanics, 2017, no.5, pp. 3840. (Rus). doi: 10.20998/2074-272X.2017.5.05.

9. Jungerman J.A. Fourth-order uniform electric field form two charged rings. Review of Scientific Instruments, 1984, vol.55, no.9, pp. 1479-1482. doi: 10.1063/1.1137962.

10. Mikhailov V.M. Raschet elektricheskikh i magnitnykh polei $s$ pomoshch'iu integral'nykh i integrodifferentsial'nykh uravnenii [Calculation of electric and magnetic fields using integral and integrodifferential equations]. Kiev, UMC VO Publ., 1988. 60 p. (Rus).

11. Ianke E., Emde F., Lesh F. Spetsial'nye funktsii [Special functions]. Moscow, Nauka Publ., 1977. 344 p. (Rus).
12. Mikhailov V.M., Chunikhin K.V. Testing of numerical solution of the problem of determining sources of magnetostatic field in magnetized medium. Electrical engineering \& electromechanics, 2017, no.6, pp. 42-46. (Rus). doi: 10.20998/2074-272X.2017.6.06.

13. Polivanov K.M. Teoreticheskie osnovy elektrotekhniki, ch. 3. Teoriia elektromagnitnogo polia [Theoretical foundations of electrical engineering, Part 3. Theory of electromagnetic field]. Moscow, Energiya Publ., 1969. 352 p. (Rus).

14. Kurbatov P.A., Arinchin S.A. Chislennyi raschet elektromagnitnykh polei [Numerical Calculation of Electromagnetic Fields]. Moscow, Energoatomizdat Publ., 1984. 168 p. (Rus).

Received 16.08.2018

K.V. Chunikhin, Postgraduate Student,

State Institution «Institute of Technical Problems of Magnetism of the NAS of Ukraine»,

19, Industrialna Str., Kharkiv, 61106, Ukraine, phone +380 572992162 ,

e-mail:kvchunikhin@gmail.com

How to cite this article:

Chunikhin K.V. On the influence of the level of an external magnetic field and the length on the magnetic moment of cylindrical cores. Electrical engineering \& electromechanics, 2018, no.6, pp. 51-55. doi: 10.20998/2074272X.2018.6.07. 